\title{
Theory of the interaction of flat sensing organ with the head of the sugar beet root
}

\author{
Volodymyr Bulgakov, ${ }^{1}$ Valerii Adamchuk, ${ }^{2}$ Ladislav Nozdrovicky, ${ }^{3}$ Ivan Holovach ${ }^{1}$ \\ ${ }^{1}$ National University of Life and Environmental Sciences of Ukraine, Kiev, Ukraine; ${ }^{2}$ National Scientific Centre, \\ Institute for Agricultural Engineering and Electrification NAAS, Ukraine, Vasylkiv District, Kiev Region, Ukraine; \\ ${ }^{3}$ Slovak University of Agriculture in Nitra, Nitra, Slovak Republic
}

\begin{abstract}
Sugar beet leaves now are very widely used for livestock feeding, as an organic fertiliser, and also as a raw material for the production of biogas. Therefore the harvest of the sugar beet tops (including leaves) can be considered as current task for the sugar beet growing system. Modern technologies involve harvest of the tops of sugar beet in two stages: flat basic cut and collecting of the entire green mass at higher altitude and the subsequent cutting of the heads of root crops from the residues. Therefore, topical issues of the sensing of the heads of sugar beet roots arranged in rows, are related to the majority of the sugar beet toppers, cleaners of the sugar beet heads, leaves cutters and, digging up working bodies of some designs. The aim of this study is theoretical determination the optimum design and kinematic parameters of a new sensing mechanism of the sugar beet heads located in the soil on the basis of the theory of interaction of flat passive swath board sensing organ with the sugar beet heads during their topping when located in the soil. In the study there are used methods of creation of mathematical models of functioning of the agricultural machines and their working bodies with the using of main provisions of mathematics, theoretical mechanics, programming and numerical calculations on the PC. In this paper, there is presented a theoretical study of the interaction of passive sensing organ with the head of the sugar beet root when there are located residues of the leaves on a root head spherical surface in the form of short elastic rods. Thus, for such an interaction of the sensing organ and the head of sugar beet root head there is taken into account elastic-damping properties of the sugar beet leaves residues. In the study there was first of all developed a new design of the topper for sugar beet heads with the use of a flat
\end{abstract}

Correspondence: Ladislav Nozdrovicky, Slovak University of Agriculture in Nitra, Tr. A. Hlinku 2, 949 76, Nitra, Slovak Republic. E-mail: ladislav.nozdrovicky@gmail.com

Key words: Sugar beet; harvest; sugar beet leaves; sensing of the heads; theory; equations of motion; the kinematic and design parameters.

Received for publication: 7 February 2017.

Accepted for publication: 3 August 2017.

(C) Copyright V. Bulgakov et al., 2017

Licensee PAGEPress, Italy

Journal of Agricultural Engineering 2017; XLVIII:676

doi:10.4081/jae.2017.676

This article is distributed under the terms of the Creative Commons Attribution Noncommercial License (by-nc 4.0) which permits any noncommercial use, distribution, and reproduction in any medium, provided the original author(s) and source are credited. swath board sensing organ, there was developed the equivalent scheme of the interaction of the sensing organ with a fixed sugar beet root head. There was also selected and arranged the axis of coordinates, and determined the active forces and angle parameters. Using the basic law of dynamics there was made up a new system of differential equations and the integration of which has made it possible to find the laws of variation of the speed of movement and of the movement of the flat passive sensing organ on the head of sugar beet root head at the beginning of the contact. After determining the initial values of force and design parameters it is possible to solve them on PC to optimise kinematic and structural parameters of the sensing mechanism.

\section{Formulation of the problem}

Modern technologies of sugar beet harvest are based on the procedure that before the digging of the sugar beet roots from the soli there is provided the cut of the leaves and subsequent final topping of the sugar beet heads at the root. Therefore, the effective functioning of the majority of sugar beet harvest machines depends on how sensitive and accurate is the sensing of sugar beet roots, located in the row, the heads of which are raised at different heights above the soil surface. This applies to most sugar beet toppers, machines for the cleaning of the heads of the sugar beet roots and sugar beet digging working bodies of some design.

In world practice, there are widespread used a varieties of mechanical sensing devices that sense the heads of the sugar beet roots located in the soil and subsequent using of topping and heads cleaning mechanisms to the required installation height. There can be used passive swath board sensing mechanisms, active mechanisms (driven-type and drum type) and also combined mechanisms. However, the requirements on the required level of work quality in a different harvest conditions, simplicity of design, lower metal consumption and energy consumption, are not always fulfilled.

\section{Analysis of recent publications}

The problems of the removing of the residual tops of sugar beet after continuous topping at the root by topping machines are analysed in many published scientific papers and theses (Pogorelij, 1964; Ogurechnikov, 1977; Mishin, 1981; Pogorelij and Tatjanko, 1983; Khelemedik, 1996; Bulgakov, 2002). There are presented the results of the research focused on cleaners of the sugar beet root heads having different design: blade type (Pogorelij, 1964; Ogurechnikov, 1977; Bulgakov, 2002), ring type (Mishin, 1981), sector type (Martynenko, 1992) and drum type (Pogorelij, 1964), as well as in the form of a paraboloid (Khelemedik, 1996). There are mostly used the cleaners of the sugar beet root heads located in the 
soil that do not require a very precise and sensitive copying each head of the sugar beet root, which are arranged in a row at various heights above the soil surface. Technological processes of harvesting the tops of sugar beet and fodder beet are similar in many respects, however, the study of harvesting of the fodder beet tops are devoted only experimental work (Mishin, 1981; Gurchenko and Savchenko, 1986). It should be emphasised that the theoretical studies of the movement of a passive comb-type sensing organ on the heads of sugar beet (especially when it is moved from root which is low-lying in the soil on a highly placed, and vice versa) are given by Gurchenko and Zavgorodnij (1987). However, the theoretical results of these studies are based mainly on the use of geometric constraints. The mathematical model of the process here is not developed.

In Bulgakov (2002) it is considered the impact interaction of the flat passive sensing organ inclined in longitudinal-vertical plane and moving steadily, with the head of the sugar beet root, which is located motionless in the ground, the head of which stands above the soil surface. However, the theory presented here, examines only a special case of such interaction, when the head of sugar beet root is high enough above the surface of the soil, devoid of any residue on the sugar beet leaves on its spherical surface and it is represented in the form of a rigid body. It should be emphasised that at the present time in the world there have been widely distributed the simplest toppers of the heads of sugar beet from the residues of the tops, containing a comb mounted in front and a flat knife mounted on the back of it, mounted on the same frame. However, as the results of comprehensive long-term tests of such design of the sugar beet root toppers, the use, in this case, of comb-shaped sensing organs, leads to the fact that short residues of the tops (stalks), as a rule, are located in intervals between the copier's combs when the copier hits the head and combs contact directly with the body of the upper part of the beetroot root, i.e. with its head.

This leads to the fact that the combs (in fact, rather narrow, located metal strips with gaps) not only cause deep traumas to the bodies of sugar beet roots, but actually establish a high level of subsequent circumcision, i.e. provide a significantly reduced height of the cut of the sugar beet root heads. This leads to the fact that it is completely unjustified when the heads of sugar beet root crops are physically lost up to $6-8 \%$ of the sugar-bearing mass, vertical cracks are formed in the upper parts of the sugar beet roots, and the losses of beet juice are more intense (Pogorelij and Tatjanko, 2004; Bulgakov, 2011).

In addition, in the case of an increase of the speed of translational motion of more than $2.0 \mathrm{~m} . \mathrm{s}^{-1}$, the use of fairly simple designs of the sugar beet toppers from the remains of the tops of the root leads to galloping, i.e. periodic jumping (especially in cases where the heads of sugar beet roots are located at different heights of protrusion above the soil surface), which leads to the fact that some heads of sugar beet roots are not cut at all.

Mentioned shortcomings and disadvantages can be eliminated by using of the sugar beet toppers having a new designs, when their schemes use flat passive flat sensing organs copiers, and all of their moving part is supplied with springs, pressing (with a given and adjustable force) a sensing organs and knives to the heads of sugar beet roots (taking into account its own weight).

\section{The aim of the study}

There is an aim theoretically to determine the optimal design and kinematic parameters of the new sensing working organ of the sugar beet topper for topping of the sugar beet root tops based on the creation of the theory of interaction of passive swath-board type sensing organ with the heads of sugar beet roots located in the soil during their topping.

\section{Research methods}

There were used the methods of developing of mathematical models of functioning of the agricultural machines and their working organs based on the general provisions of mathematics, theoretical mechanics, programming and numerical calculations on the PC.

\section{Basic results of the research}

We have developed the new design of the passive-type sugar beet heads topper used when the sugar beet roots are standing in a soil. The design and technology scheme is presented on a Figure 1.

Sugar beet heads topper used when the sugar beet roots are standing in a soil, consists of a frame 1 , which is connected to the main frame of the sugar beet topper (which is providing full cut of

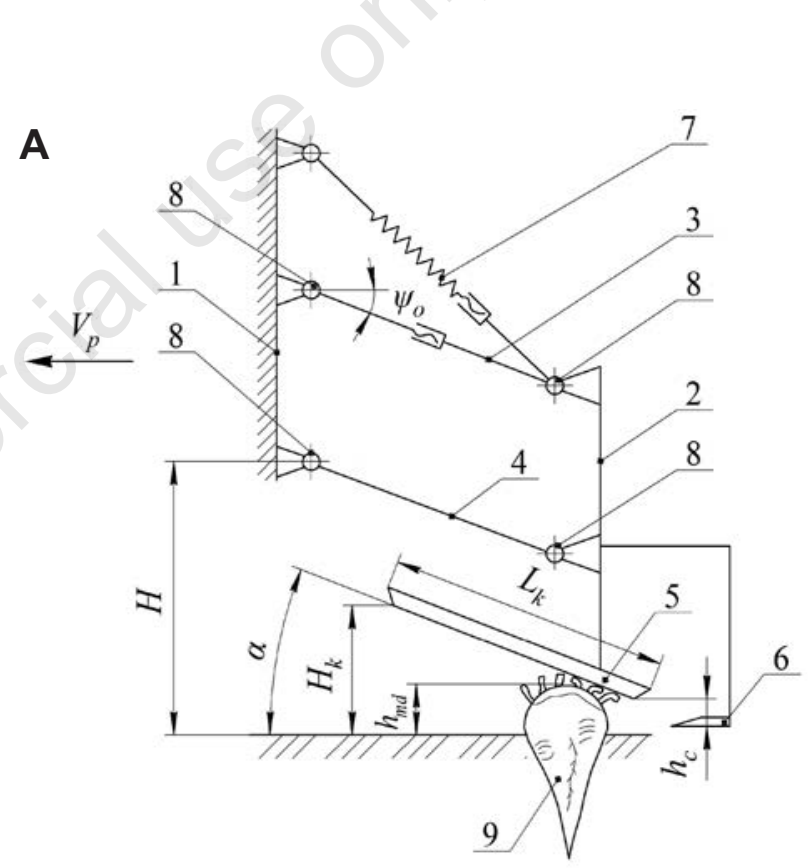

B

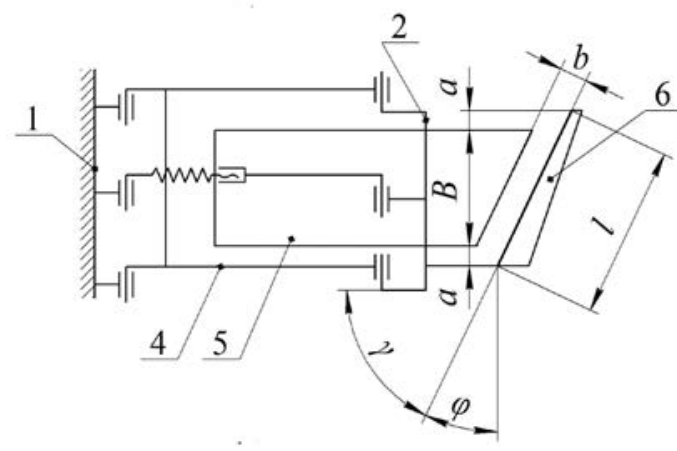

Figure 1. Design and technology scheme of the passive topper for topping the heads of the sugar beet root: A) view from the side; B) view from the top: 1 - frame; 2 - pillar with working organs; 3, 4 - rods, creating parallelogram mechanism; 5 - swath-board type sensing organ; 6 - flat knife; 7 - spring mechanism; 8 hinges; 9 - sugar beet root. 
the sugar beet tops without support) over each row of the sugar beet roots, pillar 2 with the working organs, which is connected to the frame 1 by means of two pairs of rods 3 and 4 of the parallelogram mechanism. The rod 3 has a screw mechanism allowing regulating its length, ensuring the accuracy of the setting the sugar beet topper in the longitudinal-vertical plane.

The pillar 2 in the lower part is equipped by swath-board type sensing organ 5. Its angle $\alpha$ of inclination in relation to the horizontal axis can be regulated. Behind it there is located passive knife 6, having length $l$ and it is deflected by an angle $\gamma$ to the direction of travel $\left(V_{p}\right)$. All movable parts of the sugar beet topper are interconnected by hinges 8 , and rods 3 and 4 , moreover, are connected by the spring mechanism 7. At the same time, the upper right part of the topper is connected to the stationary frame of the hitch by the spring mechanism 7, which also has a screw mechanism for adjusting its length.

Sugar beet root topper is working by the following way. While moving along the rows of the sugar beet roots [from which they were cut before, with a solid, unprimed cut, the bulk of the green foliage, but on the heads of root crops there were short remains (part of the green stalks) of the foliage], the flat passive swathboard type sensing organ 5 penetrates into the root 9 and moving in its head, lifts the pillar 2 with a knife 6 up, and when the rear part of the sensing organ reaches the top of the head, the knife 6 finally set on the desired cutting height. In subsequent forward movement of the topper knife 6 cuts the flat head from the residues of the sugar beet leaves together with the leaves residues (parts of the green stalks). The topped sugar beet root remains on the field surface.

Depending on the gaps $h_{c}$ and $b$ there occurs the change the height of cut of the head of the sugar beet root. The size of these gaps depends on the statistical sizes of sugar beet roots and it is regulated individually for each sugar beet plantations. The spring mechanism 7 is used for damping of the topper shaking of the mobile part of the topper when moving on the heads of the sugar beet having a different height, which presses the sensing organ 5 to the head of sugar beet root 9 .

Based on the statistical size-mass characteristics of sugar beet roots and their arrangement in rows of crops, some basic design parameters of the topper can be taken constructively or calculated from the known simple enough dependencies. Thus, the width $B$ of the flat sensing organ is considered within the range $160.10^{-3}$ $210.10^{-3} \mathrm{~m} ; H_{k}$ - the height of the installation of periphery of the swath-board type sensing organ 5 is considered greater than the height of location of sugar beet root 9 above the soil surface, and shall be equal to not less than $150.10^{-3}-170.10^{-3} \mathrm{~m} ; H$ - the height of the location of lower hinge 8 of a parallelogram suspension above the level of the soil surface is chosen to be constructively and should be within the range $250 \cdot 10^{-3}-350.10^{-3} \mathrm{~m}$. At the same time the value of a height $H$ must also provide the transport clearance of the sugar beet harvester machines, not less than $300.10^{-3}$ $\mathrm{m}$. The length of the flat swath-board type-sensing organ 5 depends upon the maximal height of the head of the sugar beet root 9 and can be determined by using the following equation:

$$
L_{k}=\frac{H_{k}}{\sin \alpha}
$$

The vertical gap $h_{c}$ between the knife 6 and flat swath-board type sensing organ 5 depends upon the height of location of the head of sugar beet root and it should be within the range $10.10^{-3}-$
$50.10^{-3} \mathrm{~m}$. The horizontal gap $b$ between the sensing organ 5 and knife 6 depends upon the maximal value of the diameter of the sugar beet head 9 and it is within the range $b=0-80.10^{-3} \mathrm{~m}$. The angle $\gamma$ should provide smooth motion of the topped head of the sugar beet root and others crop residues from the edge of the knife 6. It can be determined by using of the following equation:

$\gamma=90^{\circ}>\varphi$

where $\varphi$ - angle of friction of the crop material on the edge of the knife, $45^{\circ}>\varphi>40^{\circ}$.

The length $l$ of the knife 6 can be determined by the following equation:

$$
l=\frac{B+2 a}{\cos \varphi}
$$

where $a$ - overlapping of the knife 6 with the respect to the width of the sensing organ 5 , which is determined constructively.

However, the efficient operation of the given machine mechanism of a sugar beet harvester will be carried out only when the power parameters of the interaction of the flat passive swath-board sensing organ with the head of the root will satisfy the conditions of its withdrawal out of the soil during the working process and ensure a minimum of damage of them at a high forward speed movement.

In the conditions that are most similar to the real conditions, the interaction of a flat passive sensing organ in the initial time moment is based on contacts with the short and elastic tufts of sugar beet leaves, which remained on the side and the centre of the spherical surface of the head of sugar beet root after cutting the leaves by a rotary working organ.

Then, when considering just such a case of the interaction of the sensing organ and sugar beet root fixed in the soil, the proposed scheme of the contact can obtain such form (Figure 2).

Thus, in conditions closer to the real conditions, the above

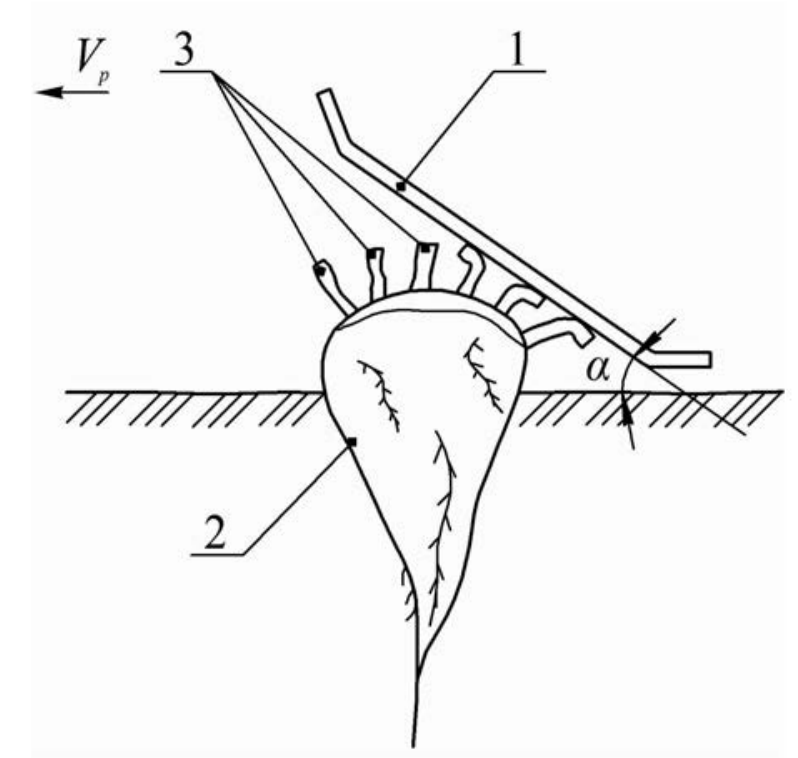

Figure 2. Interaction of the flat passive swath-board type sensing organ with the head of a sugar beet root: 1 - sensing organ; 2 sugar beet root; 3 - residues of the sugar beet leaves. 
interaction is not rigid, as it is in the case already known, and with regard to the elastic and damping properties of short residues of a green leaves, which are at the time of topping sufficiently strong.

In the next step we will make the equivalent scheme of the interaction of flat passive sensing organ, which is inclined to the horizontal axis under the angle $\alpha$, and it is moving with the forward speed $\bar{V}_{p}$ and bumping the head of the sugar beet root, initially comes into contact with elastic tops residues that are located on the side of the spherical surface of the head of sugar beet root (Figure 3).

In this case the sugar beet root is considered as a rigidly fixed with its conical part in the soil, and the most upper part of it - a head of the root acts to a certain height above the soil surface and is conventionally considered in a vertical longitudinal plane of the circle radius $R$. The centre of the head of the sugar beet root is marked as a point $O$.

The elastic and damping properties of the sugar beet leaves are shown on a equivalent scheme in the form of elastic-damping model having respectively coefficients of stiffness $c$ and damping $\mu$. Interaction of the flat sensing organ with the mentioned model of a sugar beet root and leaves residues on the sugar beet root head is done in the point $A$.

We transpose through the point $A$ a system of rectangular natural coordinates $\bar{\tau} A \bar{n}$, in which the axis $\bar{\tau}$ passes through the plane of the sensing organ at a tangent to the head of sugar beet root in the point of contact, and the axis $\bar{n}$ is directed up according to normal line to the head of sugar beet root.

We will consider all the forces that are possible as a result of the interaction of a flat sensing organ and the residues of the sugar beet tops of the head. When constructing the equivalent circuit (Figure 3 ) of the interaction of the flat passive sensing organ 5 with the head of the sugar beet root 9 , we were faced with the task of showing the main forces acting at the point $A$ of contact between the sensing organ and the head of the sugar beet root.

We arbitrarily divide them into forces that act on the head of the sugar beet root 9 from the side of the sensing organ 5, and the forces that are transferred to the sensing organ 5 from the head of the sugar beet root 9. Let us determine each of these forces, revealing the physical essence of each force and the possible directions of their action.

For the first from the given group of the forces:

$\bar{G}$ - force of the gravity of the whole sensing organ of the topper. It should immediately be emphasised that this force, which must be applied at the centre of the mass of the topper, can be transferred to the point of contact $A$ between the sensing organ 5 and the head of the sugar beet root 9 .

In this case, it is necessary to take into account the different positions of the indicated point $A$ of contact, since its position will be determined depending on the displacement of the copier 5 along the spherical surface of the head of the sugar beet root 9 . In this case, the emerging additional torque in such a parallel transfer of force $\bar{G}$ to the point $A$ from the centre of mass of the topper is, in the first approximation, neglected, considering the magnitude of such a moment as an insignificant.

Force $\bar{F}_{s r}$ - the total force that arises as a result of the appearance of a parallelogram mechanism in each hinge 8 (Figure 1) (and of such hinges 8 in the cutter design - 10) of the moments of resistance of the turns of the four links 3, 4 of the parallelogram mechanism and the pull of the spring mechanism 7 .

The direction of this total resistance force can be at an angle to the plane of the sensing organ 5 , which certainly causes the need for its representation in the form of two components - normal and tangential. In the first approximation, we consider the solution of this problem only taking into account only the normal component, which will presented in Figure 3.

Force $F_{s t}$ - which forces the copier 5 to be pressed against the head of the sugar beet root 9 due to the presence of a spring mechanism 7 (Figure 1). Given the design of this topper, when the thrust with the spring mechanism 7 is at an angle to the plane of the sensing organ 5, this force can likewise be represented in the form of two components - normal and tangential. However, in this case, in the first approximation, we will only take into account its normal component, shown in Figure 3 and coinciding with the direction of the force $\bar{F}_{s r}$.

And only the driving force $F_{s t}$ is directed toward the movement of the cutter (the direction of the translational motion is shown $-\bar{V}_{p}$ ), so in Figure 3 it is applied to the point $A$ horizontally.

From the side of the head of the sugar beetroot to the copier there are transmitted such forces:

$\bar{F}_{c}$ - the elasticity force of the sprouts of the leaves on the sugar beet tops, which can also be represented as normal and tangential components. Also, in the first approximation, we will take into account only its normal component, which arises as a result of the forces acting on the side of the sensing organ 5 .

$\bar{F}_{\mu}$ - the viscous drag force of the sprouts of the leaves on the sugar beet tops, which can also be presented in the form of normal and tangential components. In the first approximation, we will take into account only its normal component of the given force, which arises as a result of the forces acting from the side of the sensing organ 5.

Within the equivalent circuit of Figure 3 the forces $\bar{F}_{c}$ and $\bar{F}_{\mu}$ are not presented.

The frictional force $\bar{F}_{t p}$ applied to the contact point $A$ is parallel to the working speed $\bar{V}_{p}$ in the direction opposite to the sliding direction of the copier 5 over the residues of the leaves.

In the formulation of this problem, proposed by us, in spite of the fact that in a real process the flat passive roll-like sensing organ

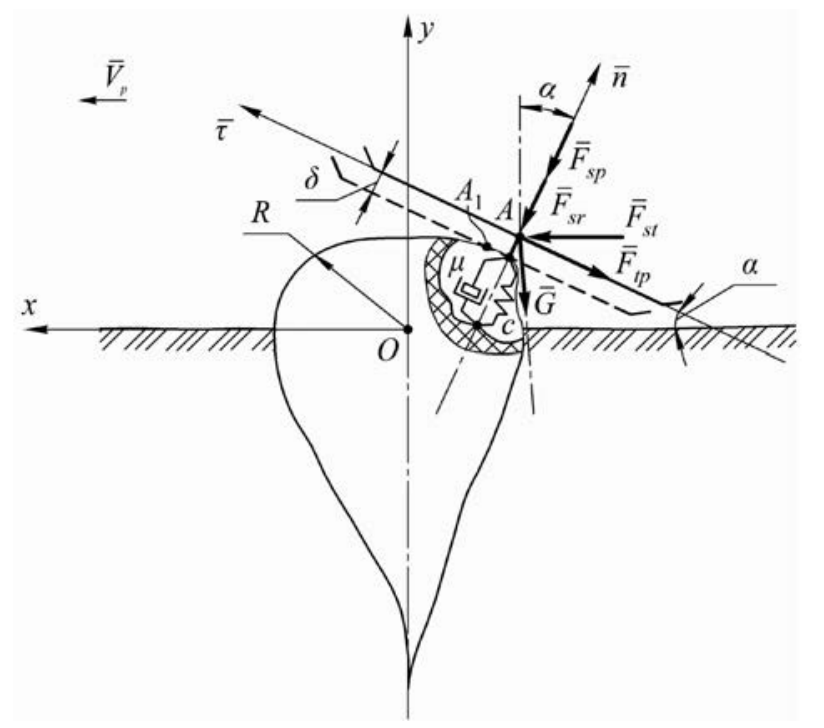

Figure 3. Equivalent scheme of an interaction of a flat passive sensing organ and head of a sugar beet root. 
5 acts (moves) not only in the normal but also in the tangential directions, we will take into account its displacement only in the normal direction by an value of the $\delta-\delta_{o}$, as such its displacement (approaching the body of the head of the sugar beet root, containing the sacchariferous mass) will determine the quality of the technological process of cutting the remains of the tops from the heads of the sugar beet root.

Since taking into account the crumpled layer of the residues of the tops to its minimum value in this direction, direct contact is excluded, and therefore the root itself is injured. The deformation of the tops in the other direction (for example, in the longitudinal direction) will not significantly affect the indicated quality of damage to the sugar beet roots.

With the regard to the resulting scheme of the forces (Figure 3), on the basis of the fundamental law of dynamics of a material point, we write the differential equation of motion of flat sensing organ on the head of sugar beet root in the vector form:

$$
m \bar{a}=\bar{F}_{s t}+\bar{F}_{s r}+\bar{F}_{s p}+\bar{F}_{c}+\bar{F}_{\mu}+\bar{F}_{t p}+\bar{G}
$$

where $\bar{a}$ - acceleration of the motion of the flat sensing organ on the head of a sugar beet root; $m$ - weight of the flat sensing organ.

The movement of the sensing organ we will consider in absolute fixed Cartesian coordinate system $x O y$ where the horizontal axis $O x$ is directed in the direction of motion of the copier, and the axis $O y$ is directed vertically upward, the centre of the coordinate system (point $O$ ) is located in the centre of the circle, the upper part of which simulates the shape of the surface of the head of the sugar beet root.

We determine the values of all the forces appearing in the vetor Equation (4). The forces of elasticity $\bar{F}_{c}$ and the viscous resistance (damping) $\bar{F}_{\mu}$ will be considered as arising from the strain $\gamma$ and strain rate $\dot{y}$ of elastic residues (roots) tops and acting in the direction normal $n$ to the surface of the head of sugar beet root.

Moreover, the deformation of the elastic residues of the sugar beet root tops largely depends on the arrangement of rootlets on the head of the sugar beet root before beginning contact with the surface of the sensing organ. Thus, at the beginning of the contact, some roots can undergo compression deformations, some - bending, some - compression and bending simultaneously.

However, once the some parts of the roots are pressed against the head of the root by the flat surface of the sensing organ, it can be assumed that this bundle of bent rootlets will subsequently undergo deformation of compression, down to partial collapse. Therefore, just this bundle of compressed roots of the residues of the leaves will create an elastic-viscous resistance to the direct contact of the flat sensing organ with the spherical surface of the sugar beet root, preventing it from damage. Obviously, in the general case, the magnitude of deformation $\gamma$ and the speed of this deformation $\dot{y}$ can depend on the coordinates $x, y$ of the position of the sensing organ, speed $\dot{x}, \dot{y}$ of the movement of the sensing organ over the head of the sugar beet root, and the time of this displacement.

Moreover, the elastic deformation of the leaves residues depends on the location of the of rootlets on the head of root crops before contact with the surface of the sensing organ. Thus, at the beginning of contact may undergo some rootlets can be effected by compression deformation, some of them by bending, and also by simultaneous bending and compression.

However, once the rootlets are pressed against the flat surface of the sensing organ to the head of sugar beet root, it can be assumed that the bunch of bent rootlets will continue to be subjected to compressive strain, until the partial collapse.

Therefore, this bunch of compressed of residues of rootlets tops will create an elastic-viscous resistance of the direct contact of flat sensing organ with the spherical surface of the head of sugar beet root, preventing it from being damaged.

Obviously, that in general, the quantity of strain $\gamma$ and strain rate $\dot{y}$ may depend on the coordinates $x, y$ of positions of the sensing organ, the sensing organ moving speed $V$ on the head of the sugar beet root and the time $t$ of this displacement. To determine this dependence theoretically is quite difficult.

Therefore, the values of these forces can usefully be determined according to the following expressions (in the beginning in general form):

$$
\begin{aligned}
& F_{c}=c \cdot \gamma(x, y, t), \\
& F_{\mu}=\mu \cdot \dot{\gamma}(x, y, \dot{x}, \dot{y}, t)
\end{aligned}
$$

where $\gamma(x, y, t), \dot{y}(x, y, \dot{x}, \dot{y}, t)$ - respectively, the magnitude of deformation and speed of deformation of the bases of leaves, while in contact with the surface of the sensing organ; $c$ - coefficient of elastic deformation of the bases of the leaves residues on the roots, $\mathrm{N} . \mathrm{m}^{-1} ; \mu$ - viscous drag coefficient (damping) of the bases of leaves on the roots, N.s.m ${ }^{-1}$.

We express the value of the strain $\gamma$ and deformation rate $\dot{y}$ of the short remains of the tops through the coordinates of the position of the sensing organ as it moves along the head of the sugar beet root at an arbitrary time.

Let in the beginning of the contact, when all the roots of the bundle of the residues of the tops that have fallen into the contact area are pressed against the head of the root by the flat surface of the sensing organ, the thickness of the formed layer from the residues of the leaves will be equal to $\delta_{0}$.

With further movement of the sensing organ over the head of the root, the indicated layer of the residues of the leaves begins to contract. Let at any time the thickness of the layer of compressed residues of the leaves be equal $\delta$. Then the deformation $\gamma$ of this layer at this instant of time will be equal to:

$\gamma=\delta=\delta_{0}$

Since the sensing organ moves on the head of the root without interruption, at any time when the sensing organ is at a point $A(x, y)$, the distance from the point $A$ to the origin of the coordinates (point $O$ ) will be equal to $\sqrt{x^{2}+y^{2}}$ and, therefore, the thickness of the layer $\delta$ expressed in terms of the coordinates of the point $A$ (the point of contact position of the sensing organ) will be equal to (Figure 4):

$$
\delta=\sqrt{x^{2}+y^{2}}-R
$$

where $R$ - radius of the head of a sugar beet root.

Taking into account expression (6), the deformation of the layer of remains of the tops at an arbitrary point $A$ will be determined by the following expression: 
$\gamma=\sqrt{x^{2}+y^{2}}-R-\delta_{o}$

Differentiating the expression (8) over time $t$, we get the value of a speed of said strain at any given time moment. We have:

$\dot{\gamma}=\frac{x \dot{x}+y \dot{y}}{\sqrt{x^{2}+y^{2}}}$

Taking into account the Equation (5), and also the Equations (8) and (9), we get the value of the elastic force of the compressed sugar beet root tops residue deformation in the following form:

$$
F_{c}=c\left(\sqrt{x^{2}+y^{2}}-R-\delta_{o}\right)
$$

and the force of viscous resistance (damping):

$$
F_{\mu}=\mu\left(\frac{x \dot{x}+y \dot{y}}{\sqrt{x^{2}+y^{2}}}\right)
$$

As it can be seen from the main scheme (Figure 3), the magnitude of the frictional force will be equal to:

$$
F_{t p}=f\left[F_{s p}+F_{s r}-F_{c}-F_{\mu}-F_{s t} \cdot \cos (\hat{x, \bar{n}})+G \cdot \cos (\hat{y, \bar{n}})\right]
$$

where $f$ - coefficient of the friction of the surface of residues of leaves on the surface of the flat passive sensing organ.

In projection on the axes $O_{x}$ and $O_{y}$ the vector Equation (4) can be written as a system of differential equations of the following form:

$$
\left.\begin{array}{rl}
m \ddot{x}= & F_{s t}-F_{s r} \cos (\hat{x, \bar{n}})-F_{s p} \cos (\hat{x, \bar{n}})+ \\
& +F_{c} \cos (\hat{x}, \bar{n})+F_{\mu} \cos (\hat{x, \bar{n}})-F_{t p} \cos (\dot{x}, \bar{V}), \\
m \ddot{y}= & -F_{s r} \cos (\hat{y, \bar{n}})-F_{s p} \cos (\hat{y, \bar{n}})+F_{c} \cos (\hat{y,} \bar{n})+ \\
& +\bar{F}_{\mu} \cos (\hat{y,} \bar{n})-\bar{F}_{t p} \cos (\hat{y}, \bar{V})-G,
\end{array}\right\}
$$

where $\cos (x, \bar{n}), \cos (y, \bar{n})$ - the direction cosines of the vector of the normal $\bar{n}$ to the axis $O_{x}$ and $O_{y}$ respectively; $\cos (\dot{x}, \bar{V}), \cos (\dot{y}, \bar{V})-$ the direction cosines of the vector of the forward speed $\bar{V}$ to the axis $O_{x}$ and $O_{y}$ respectively; $\dot{x}, \dot{y}$ - projection of the vector of the forward speed $\bar{V}$ to the axis $O_{x}$ and $O_{y}$ respectively.

According to Vasilenko (1996) the mentioned direction cosines will be equal to: $\cos (\hat{x, \bar{n}})=\frac{d f}{d x} \cdot \frac{1}{\Delta f}, \cos (\hat{y, \bar{n}})=\frac{d f}{d y} \cdot \frac{1}{\Delta f}$

$\cos (\dot{x}, \bar{V})=\frac{\dot{x}}{V}, \quad \cos (\dot{y}, \bar{V})=\frac{\dot{y}}{V}$

where $f(x, y)$ - the equation of the connection (surface, on which a material point moves); $\Delta f$ - module of the function gradient $f(x, y)$; $V$ - module of the vector of point forward speed.

Since it was initially assumed that the head of sugar beet root has a spherical shape, in the two-dimensional case the constraint equation has the following form:

$$
f(x, y)=x^{2}+y^{2}-R^{2}=0
$$

where $R$ - radius of the head of the sugar beet root.

The above equation is related to the circle with the radius $R$ having the centre $O$ in the centre of the coordinates.

According Vasilenko (1996) the module of the gradient function and module of the forward speed points, respectively, will be equal to:

$$
\begin{aligned}
& \Delta f=\sqrt{\left(\frac{\partial f}{\partial x}\right)^{2}+\left(\frac{\partial f}{\partial y}\right)^{2}} \\
& V=\sqrt{\dot{x}^{2}+\dot{y}^{2}}
\end{aligned}
$$

With regard to Equation (16), we have obtained:

$$
V=\sqrt{\dot{x}^{2}+\dot{y}^{2}}
$$

Then, according to (17), we obtain:

$$
\Delta f=\sqrt{(2 x)^{2}+(2 y)^{2}}=2 k
$$

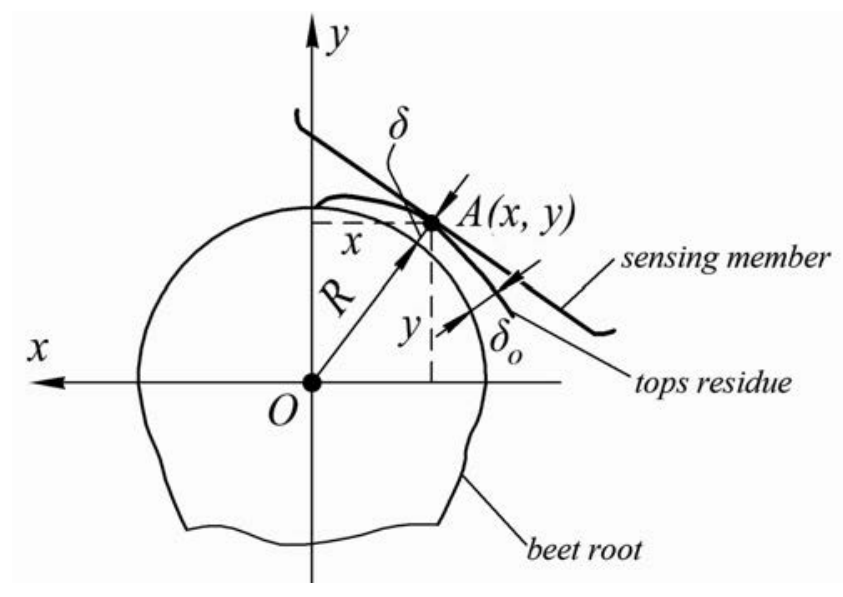

Figure 4. Scheme for determining the deformation of leaves residues on the head of sugar beet root head during contact with a flat sensing organ. 
With regard to Equations (14), (19) and (20) we have found:

$$
\cos (x, \bar{n})=\frac{x}{R}, \quad \cos (y, \bar{n})=\frac{y}{R}
$$

Taking into account (10), (11) and (21), the Equation (12) for the determination of the friction force $F_{t p}$ will be as follows:

$$
\begin{aligned}
F_{t p} & =f\left[F_{s p}+F_{s r}-c\left(\sqrt{x^{2}+y^{2}}-R-\delta_{o}\right)-\right. \\
& \left.-\mu\left(\frac{x \dot{x}+y \dot{y}}{\sqrt{x^{2}+y^{2}}}\right)-\frac{x}{R} F_{s t}+\frac{y}{R} G\right] .
\end{aligned}
$$

Substituting in (13) the Equations (10), (11), (15), (21) and (22) we obtain the following system of differential equations:

$$
\begin{aligned}
& m \ddot{x}=F_{s t}-\frac{x}{R} F_{s r}-\frac{x}{R} F_{s p}+\frac{x}{R} \cdot c\left(\sqrt{x^{2}+y^{2}}-R-\delta_{o}\right)+ \\
& +\frac{x}{R} \mu\left(\frac{x \dot{x}+y \dot{y}}{\sqrt{x^{2}+y^{2}}}\right)-\frac{\dot{x} f}{\sqrt{\dot{x}^{2}+\dot{y}^{2}}}\left[F_{s p}+F_{s r}-\right. \\
& \left.-c\left(\sqrt{x^{2}+y^{2}}-R-\delta_{o}\right)-\mu\left(\frac{x \dot{x}+y \dot{y}}{\sqrt{x^{2}+y^{2}}}\right)-\frac{x}{R} F_{s t}+\frac{y}{R} G\right] \\
& \left.\begin{array}{rl}
m \ddot{y}=\frac{y}{R} F_{s r}-\frac{y}{R} F_{s p}+\frac{y}{R} \cdot c\left(\sqrt{x^{2}+y^{2}}-R-\delta_{o}\right)+ \\
+\frac{y}{R} \mu\left(\frac{x \dot{x}+y \dot{y}}{\sqrt{x^{2}+y^{2}}}\right)-\frac{\dot{y} f}{\sqrt{\dot{x}^{2}+\dot{y}^{2}}}\left[F_{s p}+F_{s r}-c\left(\sqrt{x^{2}+y^{2}}-R-\delta_{o}\right)-\right. \\
\left.-\mu\left(\frac{x \dot{x}+y \dot{y}}{\sqrt{x^{2}+y^{2}}}\right)-\frac{x}{R} F_{s t}+\frac{y}{R} G\right]-G .
\end{array}\right\}
\end{aligned}
$$

For the purpose of calculation, the systems of differential Equations (23) can be presented in more convenient form:

$$
\left.\begin{array}{l}
\ddot{x}=\frac{F_{s t}}{m}-\frac{x}{m R}\left[c\left(\sqrt{x^{2}+y^{2}}-R-\delta_{o}\right)+\mu\left(\frac{x \dot{x}+y \dot{y}}{\sqrt{x^{2}+y^{2}}}\right)-F_{s s}-F_{s p}\right]- \\
-\frac{\dot{x} f}{\sqrt{\dot{x}^{2}+\dot{y}^{2}}}\left[F_{s r}+F_{s p}-c\left(\sqrt{x^{2}+y^{2}}-R-\delta_{o}\right)-\mu\left(\frac{x \dot{x}+y \dot{y}}{\sqrt{x^{2}+y^{2}}}\right)-\right. \\
\left.-\frac{x}{R} F_{s t}+\frac{y}{R} G\right], \\
\ddot{y}=-\frac{y}{m R}\left[c\left(\sqrt{x^{2}+y^{2}}-R-\delta_{o}\right)+\mu\left(\frac{x \dot{x}+y \dot{y}}{\sqrt{x^{2}+y^{2}}}\right)-F_{s r}-F_{s p}\right]- \\
-\frac{\dot{y} f}{\sqrt{\dot{x}^{2}+\dot{y}^{2}}}\left[F_{s r}+F_{s p}-c\left(\sqrt{x^{2}+y^{2}}-R-\delta_{o}\right)-\mu\left(\frac{x \dot{x}+y \dot{y}}{\sqrt{x^{2}+y^{2}}}\right)-\right. \\
\left.-\frac{x}{R} F_{s t}+\frac{y}{R} G\right]-g,
\end{array}\right\}
$$

where $g$ - free fall acceleration, $\mathrm{m} \cdot \mathrm{s}^{-2}$.

Obtained system of a differential Equations (24) can be accepted as a calculation mathematical model of the movement of the flat sensing organ having a permanent contact with the head of the sugar beet root. For the contact point it is very probable the occurrence of a hard impact which can always cause the damage of upper part of a sugar beet root or knocking-out of whole sugar beet root from the soil. Mentioned mathematical model in general and complex form allows to model the process of a work functioning of the passive sensing organ of a sugar beet topper with the regard of all forces, acting on a given case based on their physical character. Original mathematical model takes into account elastic-damping properties of a residues of sugar beet leaves, which undoubtedly belong to sensing system, which is used for sugar beet leaves harvest.

Besides this the obtained system of Equations (24) can be considered as a system of differential equations of a second-order, it is non-linear, and it can be solved only by numerical methods with the use of existing computer programs.

This system of differential Equations (24) can be solved by numerical methods using a personal computer, and for the particular case, also, for example, when the forces acting during operation of the dynamic system are having constant and maximum values.

Such particular case also provides an opportunity for successful determining the optimal design and kinematic parameters of the sensing organ mechanism for the topping of sugar beet roots standing in a soil to ensure the quality of its work in a wide range of operating conditions of sugar beet harvest and sugar beet field status.

The following conditions can be considered as a starting conditions in the process of solving the system of differential Equations (24):

for $t=0$ :

$$
x=x_{o}, \quad y=y_{o}, \dot{x}=V_{p}, \dot{y}=0
$$

During solution, for example, the system (24) of differential equations for some range of elastic-damping properties of residues of the sugar beet leaves tops we can get a different values of the speed of sensing organ after the initial contact with the head of the sugar beet root. The smaller is the change speed of a sensing organ after contact with a side bud of sugar beet leaves roots, there will be less impact load, the smoother and softer will be impact of a flat sensing organ on a sugar beet root. This substantially reduces the probability of knocking of the sugar beet roots from the soil and their damage, which in itself is very important for high-quality running of the technological process under consideration.

Investigating the obtained system (24) of differential equations, the similar calculations can be provided for some intervals of changing of the driving horizontal force $F_{s t}$ and the spring force $F_{s p}$ in order to evaluate the smoothness of hitting the sensing organ to the head of sugar beet root.

It is necessary to mention that the most substantial parts of the sugar beet leaves after the flat sensing-less cut they are concentrated especially on the side part of a sugar beet root heads. In a upper part of a heads of sugar beet roots mentioned residues of a leaves are less substantial, and many times they do not occur in this part. It is necessary to mention also that in the beginning of a contact they accept the basic impact between the sensing organ and sugar beet root head, which is reducing of this impact. Due to this fact, in the beginning of contact of sensing organ with the sugar beet 
root head the side buds of leaves are deformed in a large scale, creating in the same time the maximal strength and deforming forces $F_{c \max }$ and $F_{\mu \max }$, reducing an impact load, which is changed into the form of more soft interaction.

Considering the above observations, we will study the movement of a sensing organ over the head of sugar beet root in the first moment of its contact with the sugar beet root and in such starting moment the system of differential Equations (24) is significantly simplified.

Namely, in the first approximation, we can assume that the angle between the normal and the axis $O_{y}$ remains constant and equal $\alpha$, and between the tangent and the axis $O_{y}$ is equal to $\left(90^{\circ}\right.$ $-\alpha$ ), when the contact time of the sensing organ with the side part of the head of sugar beet root is very small. Then the system of differential Equations (24) obtains the following form:

$$
\left.\begin{array}{l}
\ddot{x}=\frac{F_{s t}}{m}+\left(F_{s r}+F_{s p}-F_{c \max }-F_{\mu \max }\right) \frac{\sin \alpha}{m}- \\
-\frac{f}{m} \cos \alpha\left(F_{s p}+F_{s r}-F_{c \max }-F_{\mu \max }+F_{s t} \sin \alpha+G \cos \alpha\right), \\
\ddot{y}=\left(F_{c \max }+F_{\mu \max }-F_{s r}-F_{s p}\right) \cdot \frac{\cos \alpha}{m}-\frac{f}{m} \sin \alpha\left(F_{s p}+F_{s r}-\right. \\
\left.-F_{c \max }-F_{\mu \max }+F_{s t} \sin \alpha+G \cos \alpha\right)-g .
\end{array}\right\}
$$

Thus, there was obtained a system of linear differential equations, each of which can be easily integrated in quadratures. After the first integration of the system of Equations (26) we get:

$$
\begin{aligned}
& \dot{x}=\frac{F_{s t}}{m} t+\left(F_{s r}+F_{s p}-F_{c \max }-F_{\mu \max }\right) \frac{\sin \alpha}{m} t- \\
& -\left(F_{s p}+F_{s r}-F_{c \max }-F_{\mu \max }+F_{s t} \sin \alpha+G \cos \alpha\right) \frac{f \cos \alpha}{m} t+C_{1}, \\
& \dot{y}=\left(F_{c \max }+F_{\mu \max }-F_{s r}-F_{s p}\right) \frac{\cos \alpha}{m} t-\left(F_{s p}+F_{s r}-F_{c \max }-F_{\mu \max }+\right. \\
& \left.+F_{s t} \sin \alpha+G \cos \alpha\right) \frac{f \sin \alpha}{m} t-g t+L_{1},
\end{aligned}
$$

where $C_{1}, L_{1}$ - the arbitrary constants.

After the second integration of the system of a Equation (26) we obtain:

$$
\begin{aligned}
& x=\frac{F_{s t}}{2 m} t^{2}+\left(F_{s r}+F_{s p}-F_{c \max }-F_{\mu \max }\right) \frac{\sin \alpha}{2 m} t^{2}-\left(F_{s p}+F_{s r}-\right. \\
& \left.-F_{c \max }-F_{\mu \max }+F_{s t} \sin \alpha+G \cos \alpha\right) \frac{f \cos \alpha}{2 m} t^{2}+C_{1} t+C_{2}, \\
& y=\left(F_{c \max }+F_{\mu \max }-F_{s r}-F_{s p}\right) \frac{\cos \alpha}{2 m} t^{2}-\left(F_{s p}+F_{s r}-F_{c \max }-\right. \\
& \left.-F_{\mu \max }+F_{s t} \sin \alpha+G \cos \alpha\right) \frac{f \sin \alpha}{2 m} t^{2}-\frac{g t^{2}}{2}+L_{1} t+L_{2},
\end{aligned}
$$

where $C_{2}, L_{2}$ - the arbitrary constants.

From the initial conditions (25) we obtain the values of a arbitrary constants: $C_{1}=V_{p}, L_{1}=0, C_{2}=x_{0}, L_{2}=y_{0}$.

Thus, we finally obtain:

i) the law of changes of the move of sensing organ on the head of sugar beet root at the beginning of the contact:

$$
\left.\begin{array}{l}
x=\frac{F_{s t}}{2 m} t^{2}+\left(F_{s r}+F_{s p}-F_{c \max }-F_{\mu \max }\right) \frac{\sin \alpha}{2 m} t^{2}-\left(F_{s p}+F_{s r}-\right. \\
\left.-F_{c \max }-F_{\mu \max }+F_{s t} \sin \alpha+G \cos \alpha\right) \frac{f \cos \alpha}{2 m} t^{2}+C_{1} t+C_{2}, \\
y=\left(F_{c \max }+F_{\mu \max }-F_{s r}-F_{s p}\right) \frac{\cos \alpha}{2 m} t^{2}-\left(F_{s p}+F_{s r}-F_{c \max }-\right. \\
\left.-F_{\mu \max }+F_{s t} \sin \alpha+G \cos \alpha\right) \frac{f \sin \alpha}{2 m} t^{2}-\frac{g t^{2}}{2}+L_{1} t+L_{2},
\end{array}\right\}
$$

ii) the law of movement of a sensing organ on the head of sugar beet root at the beginning of the contact:

$$
\left.\begin{array}{l}
x=\frac{F_{s t}}{2 m} t^{2}+\left(F_{s r}+F_{s p}-F_{c \max }-F_{\mu \max }\right) \frac{\sin \alpha}{2 m} t^{2}-\left(F_{s p}+F_{s r}-\right. \\
\left.-F_{c \max }-F_{\mu \max }+F_{s t} \sin \alpha+G \cos \alpha\right) \frac{f \cos \alpha}{2 m} t^{2}+V_{p} t+x_{0}, \\
y=\left(F_{c \max }+F_{\mu \max }-F_{s r}-F_{s p}\right) \frac{\cos \alpha}{2 m} t^{2}-\left(F_{s p}+F_{s r}-F_{c \max }-\right. \\
\left.-F_{\mu \max }+F_{s t} \sin \alpha+G \cos \alpha\right) \frac{f \sin \alpha}{2 m} t^{2}-\frac{g t^{2}}{2}+y_{0} .
\end{array}\right\}
$$

By varying the values of forces appearing in the expression (29), it is possible to minimise the change in speed of the sensing organ by its initial contact with the head of the sugar beet root.

For practical use of the expressions (23) and (24) we need to find a contact time $\tau$ of the sensing organ with the head of the sugar beet root. This can be done, taking into account the working speed $V_{p}$ of the topper.

We assume that on one linear meter of the sugar beet row there is not more than 6 sugar beet roots that can respond to high sugar beet yields. Next, we consider that if sugar beet head topper moves at a forward speed $V_{p}\left(\mathrm{~m} \cdot \mathrm{s}^{-1}\right)$, during the time interval $1 \mathrm{~s}$, the sugar beet topper will make contact with the $6 V_{p}$ sugar beet roots. Therefore, the contact time $\tau$ of the sensing organ with one sugar beet root will be:

$$
\tau=\frac{1}{6 V_{p}}
$$

In order not to knock out the sugar beet root of the soil during the impact of a flat passive sensing organ on the head of the sugar beet root, it is necessary to ensure the condition under which the maximum value of the horizontal component of the force $P_{\text {g.max }}$, which acts from the side of the sensing organ at the head of the sugar beet root and its allowed value $\left[P_{g}\right]$ will be determined by the following relationship:

$P_{\text {g.max }}<\left[P_{g}\right]$

We find the horizontal component of the forces acting on the head of the sugar beet root during the interaction with the sensing organ. As you can see from the scheme (Figure 3), this force will be equal to:

$$
P_{g}=F_{s t}+\left(F_{s r}+F_{s p}-F_{c \max }-F_{\mu \max }\right) \sin \alpha-\mathrm{F}_{\mathrm{tp}} \cos \alpha
$$


On the bases of (32) and (33) we can write the condition of not knocking out the sugar beet root from the soil:

$F_{s t}+\left(F_{s r}+F_{s p}-F_{c \max }-F_{\mu \max }\right) \sin \alpha-$

$-f\left(F_{s r}+F_{s p}-F_{c \max }-F_{\mu \max }+\left(F_{s t} \sin \alpha+G \cos \alpha\right) \cos \alpha<\left[P_{g}\right]\right.$

Inequality (34) can be used for testing of any set of forces incorporated in its left-hand side.

Based on results of experimental research, published in Pogorelij (1964), it can be stated that varies within the range 98 $1.15 .10^{3} \mathrm{~N}$. At value $\left[P_{g}\right]=98 \mathrm{~N}$ of soft soil (hardness of $0.5-1.0$ $\mathrm{MPa}$ ) on an average more than $45 \%$ of the sugar beet roots are knocked out. These data can be used later in the numerical calculations with the using of personal computer.

Our experimental studies (Bulgakov, 2011; Bulgakov et al., 2016) have established such values of the following coefficients: coefficient of the elasticity of the tops (short, green stems) the of sugar beet $c=1.5 \cdot 103 \mathrm{~N}^{3} \cdot \mathrm{m}^{-1}$; coefficients of the damping of the leaves (short, green stems) $\mu=18.5$ N.s.m ${ }^{-1}$; coefficient of the friction of the tops on steel $f=0.4$. The values of these coefficients have fairly stable maximum values for the sugar beet tops at the time of harvest, so during numerical simulation on a PC actually it is not necessary to vary their values to study the behaviour of a given dynamic system.

Thus, to estimate the vibration intensity of a sensing organ upon impact on the head of a sugar beet root with various design parameters of the sensing organ, it is necessary to know its speed $V$, depending on the time of contact with the beetroot root, which will be equal to:

$V=\sqrt{\dot{x}^{2}+\dot{y}^{2}}$

where $x$ and $y$ are the coordinates of the centre of the sensing organ or the law of its movement along the root of the root, determined by the expression (30).

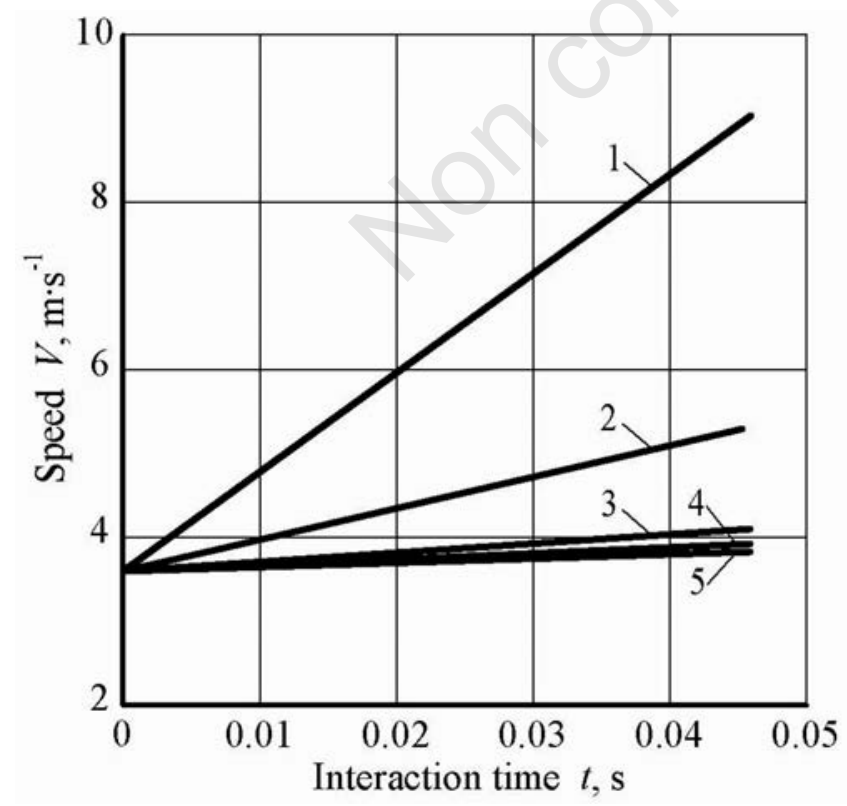

Figure 5. Dependences of the speed $V$ of the sensing organ on the contact time $t$ at an angle of inclination $\alpha=5^{\circ}$ for a different mass of the sensing organ: $1-m=1 \mathrm{~kg} ; 2-m=3 \mathrm{~kg} ; 3-m=10$ kg; $4-m=15 \mathrm{~kg} ; 5-m=20 \mathrm{~kg}$.
The results of the numerical simulation of the obtained system of equations on a PC for determining the kinematic and design parameters of the sensing organ when it moves along the head of the root crop at the translational speed $V_{p}=1 \mathrm{~m} \cdot \mathrm{s}^{-1}$ of the entire topper are presented in Figures 5-7.

From the obtained graphical dependences, presented in Figures 5-7 it can be seen that the preference should be given to curves 3 , 4 and 5 (which is close to linear dependence), which ensure minimum oscillations of the sensing organ itself in the vertical plane due to the vertical component of its speed, which plays an important role in changing the speed of the sensing organ at the beginning of its contact with the head of the sugar beet root.

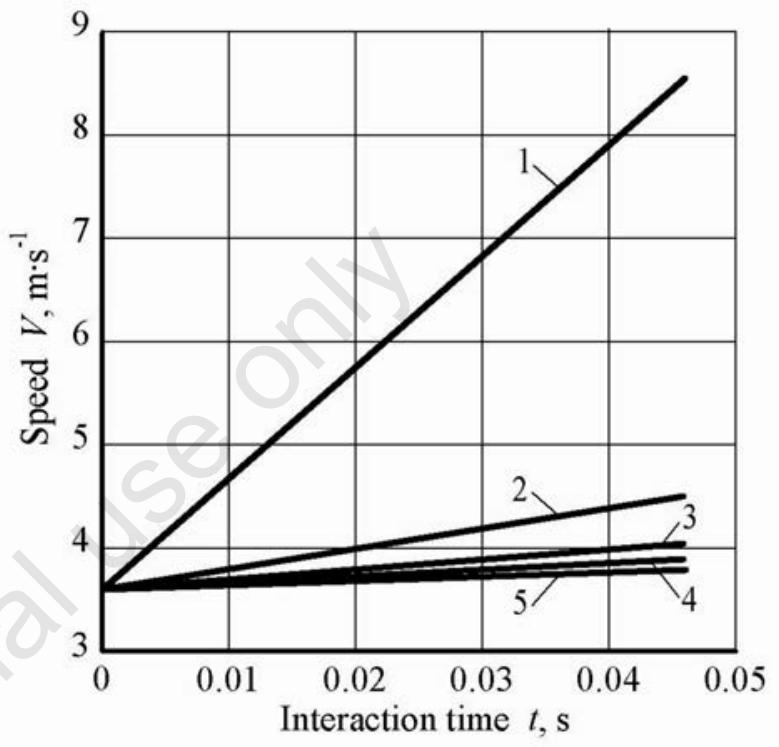

Figure 6. Dependences of the speed $V$ of the sensing organ on the contact time $t$ at an angle of inclination $\alpha=15^{\circ}$ for a different mass of the sensing organ: $1-m=1 \mathrm{~kg} ; 2-m=3 \mathrm{~kg} ; 3-m=10$ $\mathrm{kg} ; 4-m=15 \mathrm{~kg} ; 5-m=20 \mathrm{~kg}$.

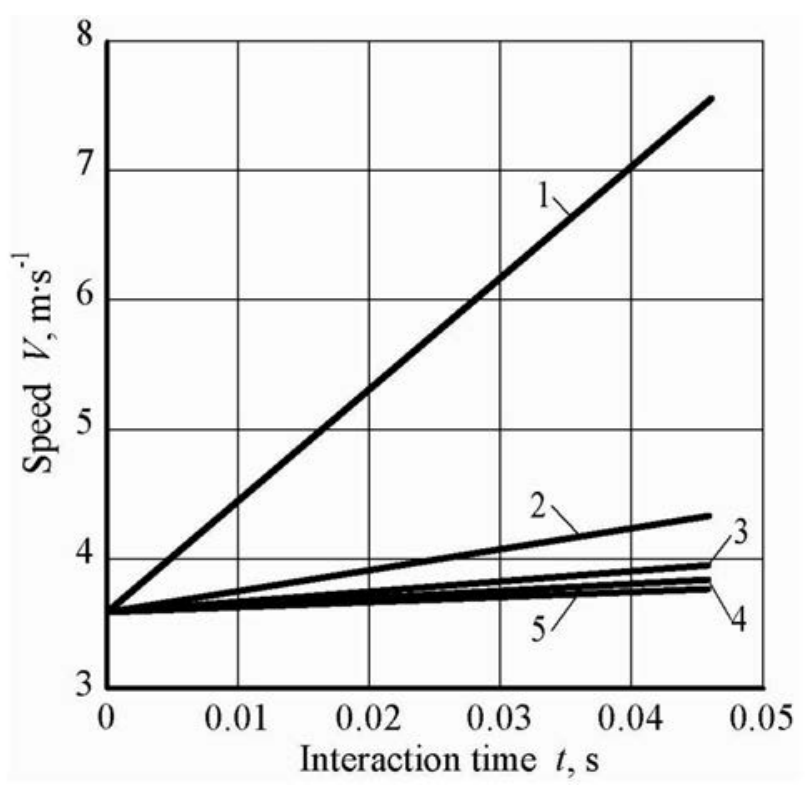

Figure 7. Dependences of the speed $V$ of the sensing organ on the contact time $t$ at an angle of inclination $\alpha=35^{\circ}$ for a different mass of the sensing organ: $1-m=1 \mathrm{~kg} ; 2-m=3 \mathrm{~kg} ; 3-m=10$ kg; $4-m=15 \mathrm{~kg} ; 5-m=20 \mathrm{~kg}$. 
Since all these three curves (3,4 and 5) are approximately on all the graphs close to each other, then, nevertheless, it is preferable to give curves 3 , which correspond to the minimum value of the sensing organ mass, equal to $m=10 \mathrm{~kg}$. One could take the mass $m$ of the sensing organ even larger, according to curves 4 and 5 (corresponding to a larger mass of the sensing organ: $4-m=15$ $\mathrm{kg} ; 5-m=20 \mathrm{~kg}$ ), but there is no reason to intentionally increase the mass of the sensing organ, due to reasons of unnecessary increase in the metal capacity of the sensing device and the topper in general.

In addition, giving preference to curve 3, we get the minimum shock load during the initial contact of the sensing organ with the head of the sugar beet root during its after-trimming. This follows from the theorem on the change in the momentum for an impact of this type (Butenin et al., 1985):

$$
m\left(\bar{V}-\bar{V}_{p}\right)=\bar{S}
$$

where $\bar{S}$ - impact impulse; $\bar{V}_{p}$ - speed of the sensing organ before the impact; $\bar{V}$ - speed of the sensing organ after the impact.

Since on all presented graphs (Figures 5-7) for curves 3, 4 and 5 , the speed differences $\bar{V}-\bar{V}_{p}$ are approximately the same (these curves are close enough to each other), then for masses $m=10 \mathrm{~kg}$, $m=15 \mathrm{~kg}$ and $m=20 \mathrm{~kg}$, we get on the basis of expression (36), an obvious inequality of the following kind:

$$
S_{\min }=10\left(\bar{V}-\bar{V}_{p}\right)<15\left(\bar{V}-\bar{V}_{p}\right)<20\left(\bar{V}-\bar{V}_{p}\right)
$$

Thus, the minimum shock pulse for curves 3 will be much smaller than for curves 4 and 5 . The use of curves 3 for further calculations will ensure that the sugar beet roots are unobstructed from the soil, when interacting with the sensing organ and excludes damage to their upper parts of the heads.

Similarly it affects the oscillations of the sensing organ and an increase in its angle of inclination relative to the direction of motion. In this case, preference should be given to large values of the angle $\alpha$, i.e. $\alpha>15^{\circ}$, as the values of the speed for the same moments of time, as seen from Figures 5 and 6 , are smaller at $\alpha>15^{\circ}$.

\section{Conclusions}

- There was developed a new design of the topper for topping the heads of the sugar beet roots standing in the soil. The experimental research and production tests have shown positive results, confirming its efficient work and high quality of the topping the heads of the sugar beet roots.

- There was developed also a new theory of the interaction of passive sensing organ with the head of the sugar beet root. For this purpose there was designed a force scheme of the interaction of a passive sensing organ and the spherical surface of the head of the sugar beet root. During this contact residues there are accounted the elastic-damping properties of the residues of the tops of the head of a sugar beet root, which are presented as model with elastic and viscous properties. At the points of contact on these schemes there are applied at the same time all the existing forces. The coordinate axes, appropriately arranged, are selected.

- By using the basic law of dynamics there was made up a new system of differential equations that describes the motion of a plane passive sensing organ on the spherical surface of the head of the sugar beet root, which contains the remains of the tops. After double integration there were obtained laws of a changes the speed of movement of the moving flat passive sensing organ on the head of sugar beet root at the beginning of the contact. There was taking into account the condition of not knocking the bodies of the sugar beet root from the soil.

- Using of a obtained new analytical dependences and the results of specific numerical calculations on the PC in the development and design of sugar beet harvesters having a modern technical level it is necessary to use the weight $m$ of the sensing organ up to $10 \mathrm{~kg}$ and the angle of its initial inclination $\alpha>15$, and it will provide a significant improvement in the quality of sugar beet tops and roots during their mechanised harvesting.

\section{References}

Bulgakov V. 2002. Study on the interaction of feeler and roots within the topping process of sugar beet. Bull. Transylv. Univ. Braşov. Series A. 9:79-84.

Bulgakov V.M. 2011. Sveklouborochnye mashiny. Agrarnaja nauka, Kiev, Ukraine [In Russian].

Bulgakov V., Adamchuk V., Nozdrovicky L. 2016. Properties of the sugar beet tops during the harvest. pp 102-108 in Proceeding of $6^{\text {th }}$ International Conference on Trends in Agricultural Engineering, 7-9 September, Prague, Czech Republic.

Butenin N.V., Lunc Ja.L., Merkin D.R. 1985. Kurs tekhnicheskoj mekhaniky. Tom 2. Nauka, Moscow, Russia [In Russian].

Gurchenko A.P., Savchenko, Ja.V. 1986. Mekhanizacija uborky botvy sakharnoj svekly. Zhurnal: Tekhnika v selskom khozjajstve. 9:15-7 [In Russian].

Gurchenko A.P., Zavgorodnij A.F. 1987. i dr. Chem ubirat botvu kormovoj svekly. Zhurnal: Mekhanizacija selskogo khozjajstva. 8:24-25 [In Russian].

Khelemedik N.M. 1996. Povyshenie mekhaniko-tekhnologicheskoj effektivnosti processov v sveklovodstve. Avtoreferat dis. DrSc. tekhn. nauk. TPI, Ternopol, Ukraine. [In Russian].

Martynenko V.Ja. 1992. Razrabotka konstrukcij i opredelenie ekspluatacionnykh parametrov ochistitelej golovok korneplodov. Avtoreferat thesis PhD. tekhn. nauk. TPI, Ternopol, Ukraine [In Russian].

Mishin M.A. 1981. Issledovanie i obosnovanie parametrov rabpchikh organov dlja doochistki golovok kornej sakharnoj svekly ot ostatkov botvy. Avtoreferat thesis PhD. tekhn. nauk. VISKhOM, Moscow, Russia [In Russian].

Ogurechnikov N.A. 1977. Izyskanie, issledoavnie i obosnovanie tekhnologicheskogo processa i rabochikh organov dlja ochistki golovok sakharnoj svekly. Avtoreferat thesis PhD. tekhn. nauk. CNIIMESKh, Minsk, Belarus [In Russian].

Pogorelij L.V. 1964. Issledoavnie i razrabotka tekhnologicheskogo processa otdelenija botvy ot kornej sakharnoj svekly. Avtoreferat thesis PhD. tekhn. nauk. USKhA, Kiev, Ukraine [In Russian].

Pogorelij L.V., Tatjanko N.V. 1983. i dr. Sveklouborochnye mashiny. Tekhnika, Kiev, Ukraine [In Russian].

Pogorelij L.V., Tatjanko N.V. 2004. Sveklouborochnye mashiny: Istorija, konstrukcija, prognoz. Feniks, Kiev, Ukraine [In Russian].

Vasilenko P.M. 1996. Vvedenie v zemledelcheskuju mekhaniku. Selkhozobrazovanie, Kiev, Ukraine [In Russian]. 